\title{
Low-loss gratings for next-generation gravitational wave detectors
}

\author{
T. Clausnitzer ${ }^{* a}$, E. -B. Kley ${ }^{\mathrm{a}}$, A. Tünnermann ${ }^{\mathrm{a}}$, A. Bunkowski $^{\mathrm{b}}$, O. Burmeister ${ }^{\mathrm{b}}$, K. Danzmann $^{\mathrm{b}}$, R. \\ Schnabel $^{\mathrm{b}}$, A. Duparré ${ }^{\mathrm{c}}, \mathrm{S}$. Gliech $^{\mathrm{c}}$ \\ ${ }^{a}$ Institut für Angewandte Physik, Friedrich-Schiller Universität, Max-Wien Platz 1, 07743 Jena, Germany; \\ ${ }^{\mathrm{b}}$ Max-Planck-Institut für Gravitationsphysik and Institut für Atom- und Molekülphysik, Universität \\ Hannover, Callinstrasse 38, 30167 Hannover, Germany; \\ ${ }^{c}$ Fraunhofer-Institut für Angewandte Optik und Feinmechanik, Albert-Einstein-Str. 7, 07745 Jena, Germany
}

\begin{abstract}
By combining electron beam lithography with coating processes, very shallow gratings with diffraction efficiencies between $0.02 \%$ and $7 \%$ have been realized. Advantages and disadvantages of different layout concepts for such gratings, their fabrication regime and measurements of their scattering will be discussed. The application of such gratings as coupling components to a reflective Fabry-Perot cavity resulted in a finesse of more than 400 .
\end{abstract}

Keywords: Diffraction gratings, gravitational wave detection, interferometry, beam splitters

\section{INTRODUCTION}

High efficiency dielectric reflection gratings composed of a highly-reflective dielectric multilayer and a grating on top have shown their excellent performance in high-power laser applications like chirped pulse amplification [1]. But lower diffraction efficiencies could also be a key to open new applications for such gratings. A very challenging field is gravitational wave detection. Today's gravitational wave detectors (for example GEO600 [2], LIGO [3]) are based on Michelson Interferometers that use methods like power and signal recycling as well as additional cavities in the interferometer arms to increase the sensitivity. The circulating laser power is in the range of a few kilowatts. However, to further increase the sensitivity of future detectors, the circulating power will be increased up to the megawatts level, where thermal effects (thermal lensing, noise) in the transmitting optics will be a limiting factor. New concepts to overcome these limitations use reflection gratings to substitute the beam splitter or the cavity coupling mirrors [4]. Recently we have demonstrated the realization of a high-finesse cavity using low-efficiency dielectric reflection gratings as cavity coupling mirrors [5]. The used gratings exhibit a diffraction efficiency in the range of $1 \%$ together with extremely low losses (scattering and transmission) and a very accurate wavefront. In the presented paper, the grating devices are discussed in more detail and it will be shown how their diffraction efficiency as well as scattering losses can be controlled.

\section{CAVITY SETUP AND GENERAL SAMPLE LAYOUT}

One setup to couple light to a cavity is illustrated in Fig. 1. The grating is illuminated in $-2^{\text {nd }}$ order Littrow mounting, where the $-1{ }^{\text {st }}$ diffraction order propagates normal to the grating (Fig. 1a). The cavity consists of the grating and a mirror that retro reflects this very weakly efficient diffraction order (Fig. 1b) (a theoretical description of the concept is given in ref. [6]). By using this setup, the finesse of the cavity is determined by the reflectivity of the mirror and the grating in case of normal incidence $R_{0^{\circ}}$. Assuming that the properties of the second cavity mirror are well known, the focus will be on the grating device here. Its reflectivity $R_{0^{\circ}}$ is influenced by the diffraction efficiencies $\eta_{1}$ of the two first orders (the efficiencies of both orders are equal if a symmetrical groove profile is assumed) as well as by losses, which includes in particular the transmission through the substrate $T_{0^{\circ}}$ (all the transmitted diffraction orders) and the scattering $S$. The reflectivity is therefore given by:

$$
R_{0^{\circ}}=1-\left(2 \eta_{1}+T+S\right) .
$$

* clausnitzer@iap.uni-jena.de; phone +49 3641 657662; fax +49 3641 657680; www.iap.uni-jena.de

Advances in Thin-Film Coatings for Optical Applications II, edited by Michael L. Fulton, Jennifer D. T. Kruschwitz, Proc. of SPIE Vol. 5870, 58700J, (2005) - 0277-786X/05/\$15 - doi: 10.1117/12.617334 
Several layouts, which are capable to realize an all-dielectric reflection grating device have been discussed previously (see for example ref. [7, 8, 9]). Most of them include a substrate, a dielectric multilayer stack and the grating itself. Grating and layerstack can be combined in two ways: firstly, the grating can be etched directly into the substrate and afterwards coated by the layerstack; secondly, it can be etched into the layerstack, which has been coated onto the substrate before. Both layouts differ in their scattering characteristics and transmission. Their particular advantages will be discussed in the next sections.

Our general goal is a high power build-up in the cavity, which requires reduction of the transmission $T$ as well as the scattering losses $S$. The finesse of the cavity is determined by the value of $R_{0^{\circ}}$. Hence, for fixed values of $\mathrm{S}$ and $\mathrm{T}$, the finesse can be controlled by changing $\eta_{l}$. For the devices discussed above, the aim was to realize efficiencies in the range below $5 \%$.

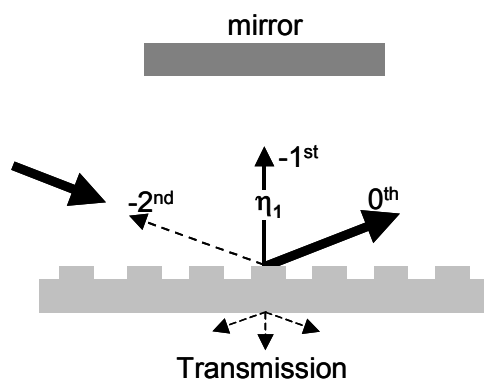

Figure 1a: Incidence from $2^{\text {nd }}$ order Littrow angle.

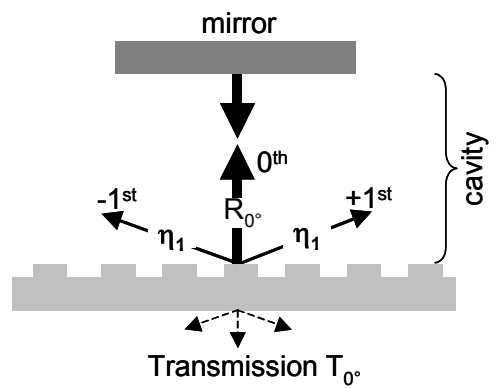

Figure 1b: Reflection for normal incidence. The transmission $\mathrm{T}_{0^{\circ}}$ is defined as the sum of all transmitted diffraction orders for normal incidence.

The cavity setup shown in Fig. 1a demands that the $-1^{\text {st }}$ order propagates normal to the grating. This means - according to the well-known grating equation - that the grating has to be illuminated under an angle that fulfills the condition

$$
\sin \varphi_{i n}=\frac{\lambda}{d} .
$$

The devices are designed for a wavelength of $1064 \mathrm{~nm}$ (Nd:YAG-laser). A period of $1.45 \mu \mathrm{m}$ was chosen in ref. [5] (this period ensures no higher propagating orders in air as well as in fused silica), so the angle of incidence has to be $47.1^{\circ}$. After the $-1^{\text {st }}$ diffraction order is retro reflected by the second cavity mirror, the light is now incident normal and the appearing diffraction orders possess the same diffraction angles as in the first case. The layerstack that is employed to avoid transmission through the substrate has to be highly reflective for normal incidence as well as for the angle determined by equation (2). The multilayer stack used here is combined of 17 layer pairs of $193 \mathrm{~nm} \mathrm{SiO}_{2}$ (refractive index $\mathrm{n}=1.45)$ and $137 \mathrm{~nm} \mathrm{Ta}_{2} \mathrm{O}_{5}(\mathrm{n}=2.02)$. The measured reflectivity of this stack is higher than $99.95 \%$ from $0^{\circ}$ incidence up to an angle of $70^{\circ}$.

\section{GRATING ON TOP OF THE HR-STACK}

The most common way to combine grating and layerstack is to etch the grating into the topmost layer of the stack. In this case, the layerstack can be designed separately, and its performance is not disturbed by the grating. Once the stack parameters are fixed, the thickness of the topmost layer of the stack and the groove depth of the grating (the groove to ridge ratio is assumed to be 1:1) have to be designed. Fig. 2 shows the diffraction efficiency $\eta_{1}$ for TE-polarized light as a function of the groove depth and the residual uncorrugated part of the topmost layer in the case it is fused silica (Fig. 2a) or tantalum pentoxide (Fig. 2b). These and all the further theoretical calculations have been done using the Fourier Modal Method ([10]). In Fig. $2 b$ the pattern is much more fluctuating. This is caused by the high refractive index and by higher diffraction orders (except for the $-1^{\text {st }}$ and $-2^{\text {nd }}$ also the $+1^{\text {st }}$ and $-3^{\text {rd }}$ order are propagating) that are guided by the topmost layer and cause resonant interference effects [9]. If the last layer is made of fused silica these higher orders do not exist. Furthermore, the etching of fused silica is an established technique, so we decided to use fused silica. The 
darker areas in Fig. $2 \mathrm{a}$ - separated by the dashed line - mark the parameters where the diffraction efficiency $\eta_{1}$ is lower than $5 \%$. If, for example, a $590 \mathrm{~nm}$ thick layer of $\mathrm{SiO}_{2}$ is chosen as the topmost layer of the stack (represented by the solid line), the diffraction efficiency will be $2.5 \% \pm 1 \%$ for groove depths between $180 \mathrm{~nm}$ and $360 \mathrm{~nm}$. Such a large tolerance is really desirable for the grating-etching step.

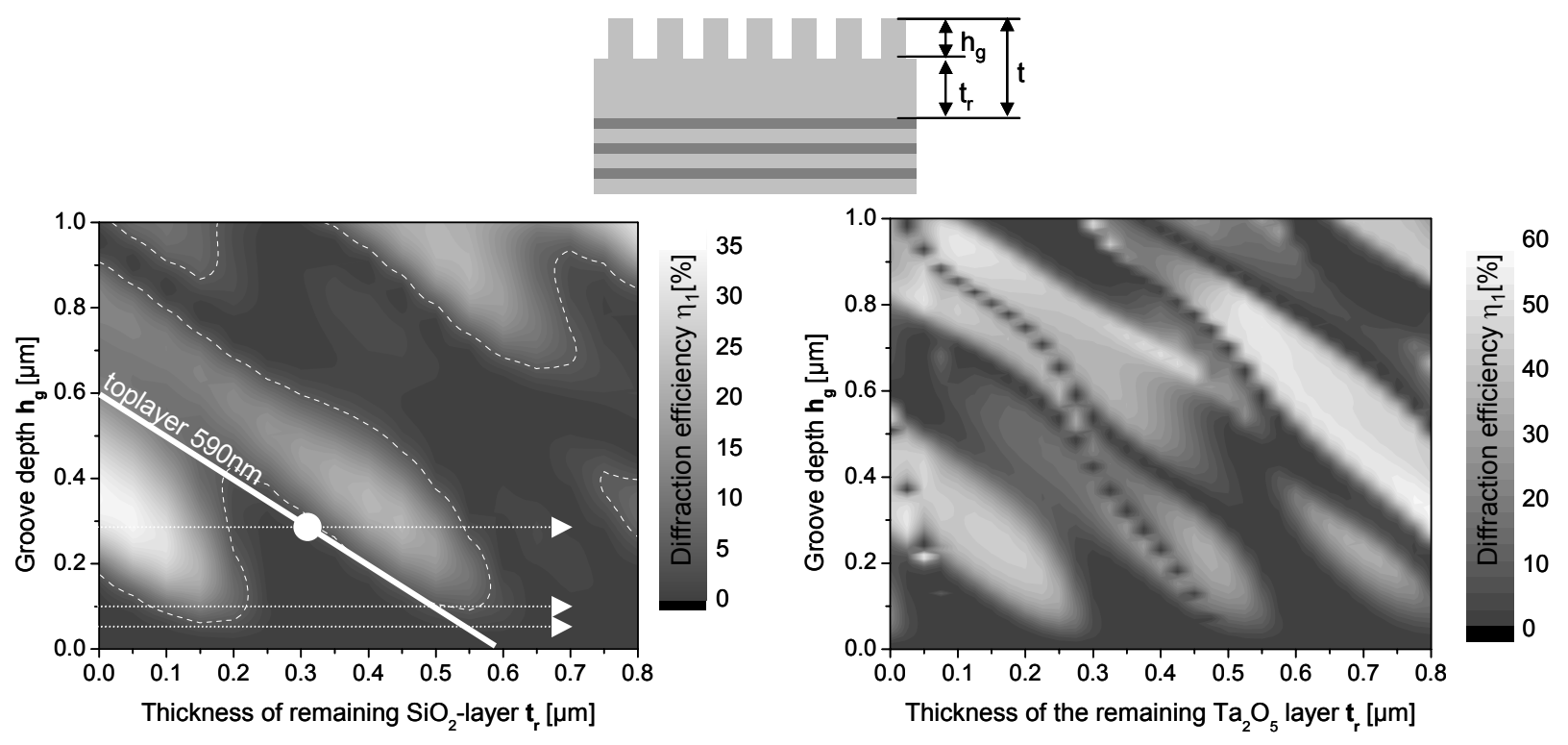

Figure 2a: Theoretical calculation of the diffraction efficiency as a function of the grating groove depth and the $\mathrm{SiO}_{2}$-layer that is remaining after the etching. The horizontal arrows are explained

Figure 2b: Like Fig. 2a but with Ta2O5 as the last layer. in section 5 .

The fabrication of the device has been done by first coating a fused silica substrate with the HR-stack by ion beam sputtering. According to Fig. 2a, a 590nm thick layer of fused silica has been coated on top of this stack. The grating pattern has been generated by electron-beam lithography and etched into the last layer by reactive ion beam etching. A groove depth of $290 \mathrm{~nm}$ and a fill factor of 0.5 were measured by an atomic force microscope (AFM). If the grating had ideal rectangular grooves and no scattering losses, it would theoretically posses a diffraction efficiency of $\eta_{1}=1.5 \%$ and thus a reflectivity of $R_{0^{\circ}}=97 \%$ [according to Eq. (1)]. To analyze the losses of the real gratings, we measured the angleresolved scattering (ARS) using the high-sensitivity ARS-instrumentation described in [11]. These measurements, of course, do not provide the value of the total scattering $S$ of the device. Estimation of total scattering by either direct measurement with an integrating or a Coblentz sphere or by calculation from measured ARS curves still raises several problems, which are a topic of further detailed studies. These investigations address the question how to estimate and even how to define the total scattering for a grating - and in particular the value of the angle separating efficiency from scatter - in harmony with the instructions given for plane surfaces in the international standard ISO 13696. However, the ARS-measurements give insight into the scattering processes and are a good measure for $S$. The samples were illuminated by a Nd:YAG laser at $1064 \mathrm{~nm}$ wavelength, TE- polarization, and normal incidence. Figure 3 shows the results of these measurements. A first order diffraction efficiency of $1.5 \%$ was also measured by a calibrated integrating sphere. Besides the expected peaks, there are some additional diffraction peaks in the range of $10^{-6}$ of the incident intensity between the $0^{\text {th }}$ and the two first orders. These orders are a result of periodic fill factor variations that are typical for e-beam lithographic pattern generation [12]. The scattering reveals a largely uniform decay over the whole space surrounding the grating, which is an indication for statistical roughness-induced scattering [13]. 

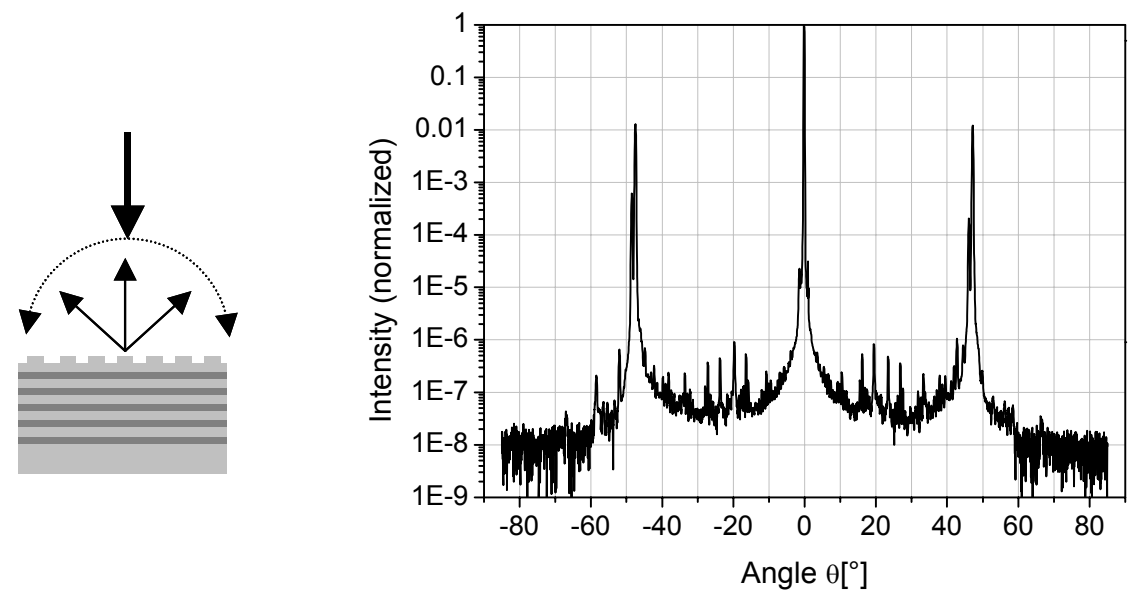

Figure 3: Angle resolved scattering measurement of a grating on top of the multilayer

\section{GRATING BENEATH THE HR-STACK}

The purpose of the device is to distribute the energy of the impinging wave into defined diffraction orders. Independent from the groove profile, these directions are only determined by the period of the grating. So the losses described in the previous section might be reduced by low-pass filtering the grating structure, as long as the period information is retained. This filtering means that all high-frequency parts of the groove profile (statistical roughness as well as the groove shape characterized by fill factor and groove depth) are smoothed. It is well known that dielectric coatings can smooth a profile [14]. Consequently, covering a grating structure with a dielectric layer is a possible way to remove high frequency perturbations like roughness or sharp edges. A smoothing of the grating profile can be achieved if the grating is first fabricated on a substrate and then coated with the HR-stack. This approach has been experimentally used to realize all-dielectric high efficiency reflection gratings [8] or low absorption low efficiency grating beam samplers [15]. A detailed theoretical analysis of this layout was done, for example, by Elson [16]. However, simulation of the diffraction efficiencies of such a device is much more complicated than in the previous approach because the layer growth on a structured substrate has to be included. We therefore investigated these elements empirically. One important issue to be considered here is the deformation of the layerstack by the grating. When the grating is on top, the layerstack is not disturbed by the grating; its reflectivity can be optimized like a simple laser mirror. Here, the surface relief of the grating influences the layer growth, so it will have a modified -and certainly increased- transmission. On the other hand, the number of layers and the resulting profile smoothing form a volume grating with variable corrugation depending on the penetration depth of the light and the shape of the grating beneath. Thus, by changing the profile of the primary grating, the diffraction efficiency of the grating device, its transmission, and its scattering can be influenced or tuned. To investigate this issue we placed gratings with several fill factors on one substrate and covered them with standard HR-layerstacks as used in the grating-on-top concept.

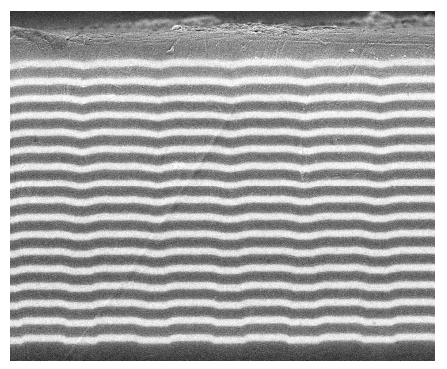

a. fill factor 0.52 , original groove depth $\mathrm{h}=40 \mathrm{~nm}$

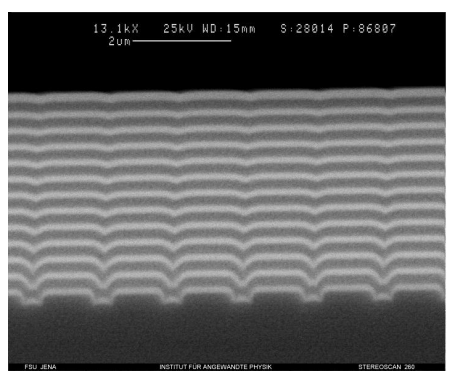

Figure 4: SEM-images of coated gratings with

b. fill factor 0.67 , original groove depth $\mathrm{h}=150 \mathrm{~nm}$

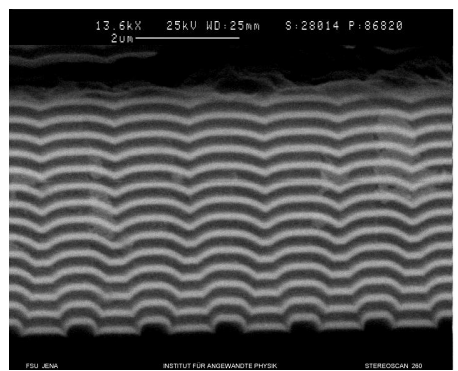

c. fill factor 0.33 , original groove depth $\mathrm{h}=150 \mathrm{~nm}$ 
In Fig. 4 the cross-section of several gratings with a groove depth of $40 \mathrm{~nm}$ and $150 \mathrm{~nm}$ (measured before the coating process by AFM) is illustrated. The corrugation depth of the volume grating is obviously decreased by increasing the number of layers. Small fill factors and therefore narrow grating ridges disturb the layerstack more than larger fill factors. However, if Fig. 4a and Fig. 4b are compared, the surface reliefs become nearly equal after a certain number of layers, independent from the depth of the original grating. It is therefore likely that the coating also smooths roughness and sharp edges.

We measured $\eta_{1}$ as well as the $0^{\text {th }}$ transmitted order with an integrating sphere. This value is a good estimation for the whole transmission of the sample as long as the diffraction efficiency is weak. The measurement results are shown in Fig. $5 \mathrm{a}$ and $\mathrm{b}$. The dashed line in Fig. $5 \mathrm{~b}$ indicates the transmission of the substrate without perturbation by the grating. The measurements confirm quantitatively what is likely from the profile considerations: a smaller fill factor causes a larger corrugation of the volume grating, resulting in higher diffraction efficiency and transmission. For larger fill factors the corrugation is smoother, the diffraction efficiency approaches zero, and the transmission becomes comparable to an undisturbed mirror. Furthermore, the graphs for the $150 \mathrm{~nm}$-deep gratings approach those of the $40 \mathrm{~nm}-$ deep gratings if the fill factor increases. This fact confirms the observations already made in the SEM-images. To quantify the scattering losses, ARS-measurements were again performed for selected gratings.

Figure 6 shows the measurement results of two gratings with a depth of $40 \mathrm{~nm}$ and fill factors of 0.49 and 0.83 (illustrated by hollow circles in Fig. 5a). In Fig. 6a, the intensity of the two first orders is nearly the same as in Fig. 3. The optical function is thus the same for both gratings. The scattered light, however, is significantly reduced. There is only one parasitic diffraction order with an intensity of $10^{-6}$ between the $0^{\text {th }}$ and the two first orders; the other peaks are in the range of $10^{-7}$ or less, while in Fig. 3 all parasitic orders are higher than $5 \times 10^{-7}$. Also, the background signal caused by statistical roughness is reduced from $3 \cdot 10^{-8}$ to $1 \cdot 10^{-8}$. In Fig. $6 \mathrm{~b}$, the scattering is further decreased, and only one parasitic order could be resolved by the measurement setup. Therefore the coating smooths not only the statistical errors but also periodical variations of the fill factor.

It can be concluded from these measurements that scattering in diffractive cavity couplers can be efficiently reduced by placing a thick dielectric layerstack on top of the grating. The resulting surface profile looks very similar for all the profiles considered here; roughness, sharp edges, and even small periodic fill factor variations are suppressed. The transmission is slightly enhanced compared to the undisturbed mirror.

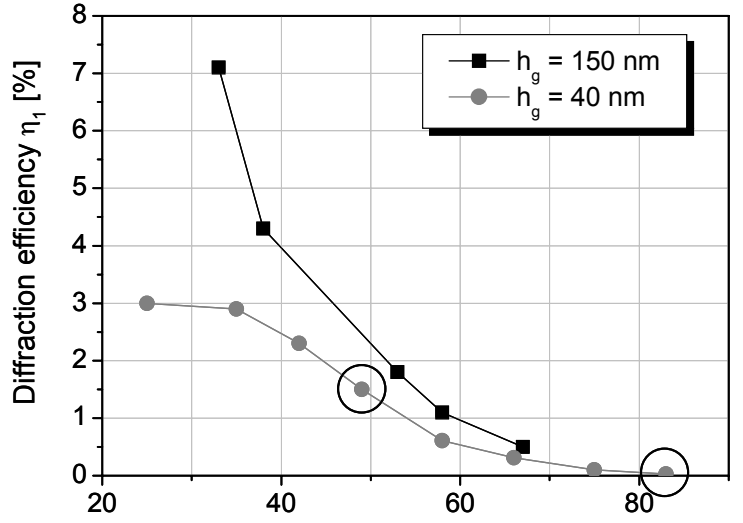

(a)

Fill factor [\%]

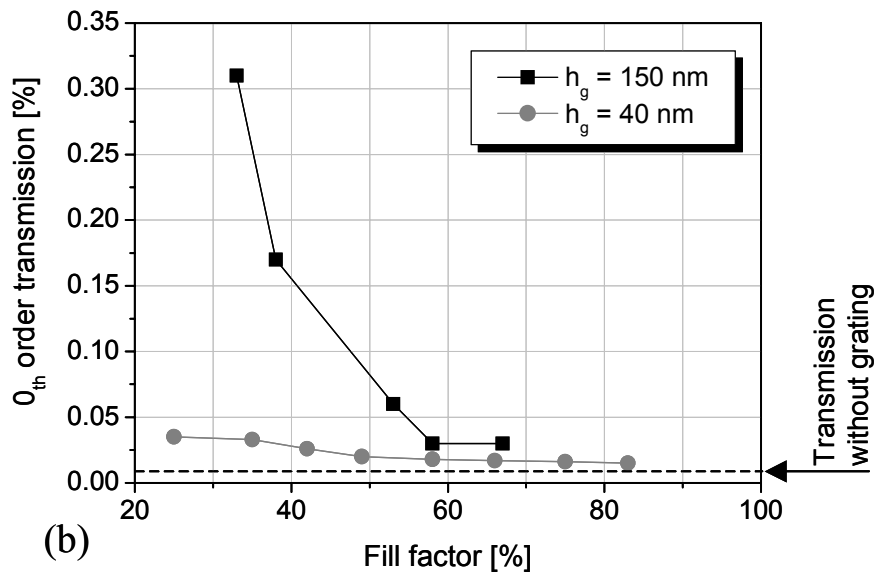

Fill factor $[\%]$

Figure 5: (a) Measured diffraction efficiency and (b) $0^{\circ}$ Transmission $\mathrm{T}_{0^{\circ}}$ as a function of the fill factor for $150 \mathrm{~nm}$ and $40 \mathrm{~nm}$ groove depth 

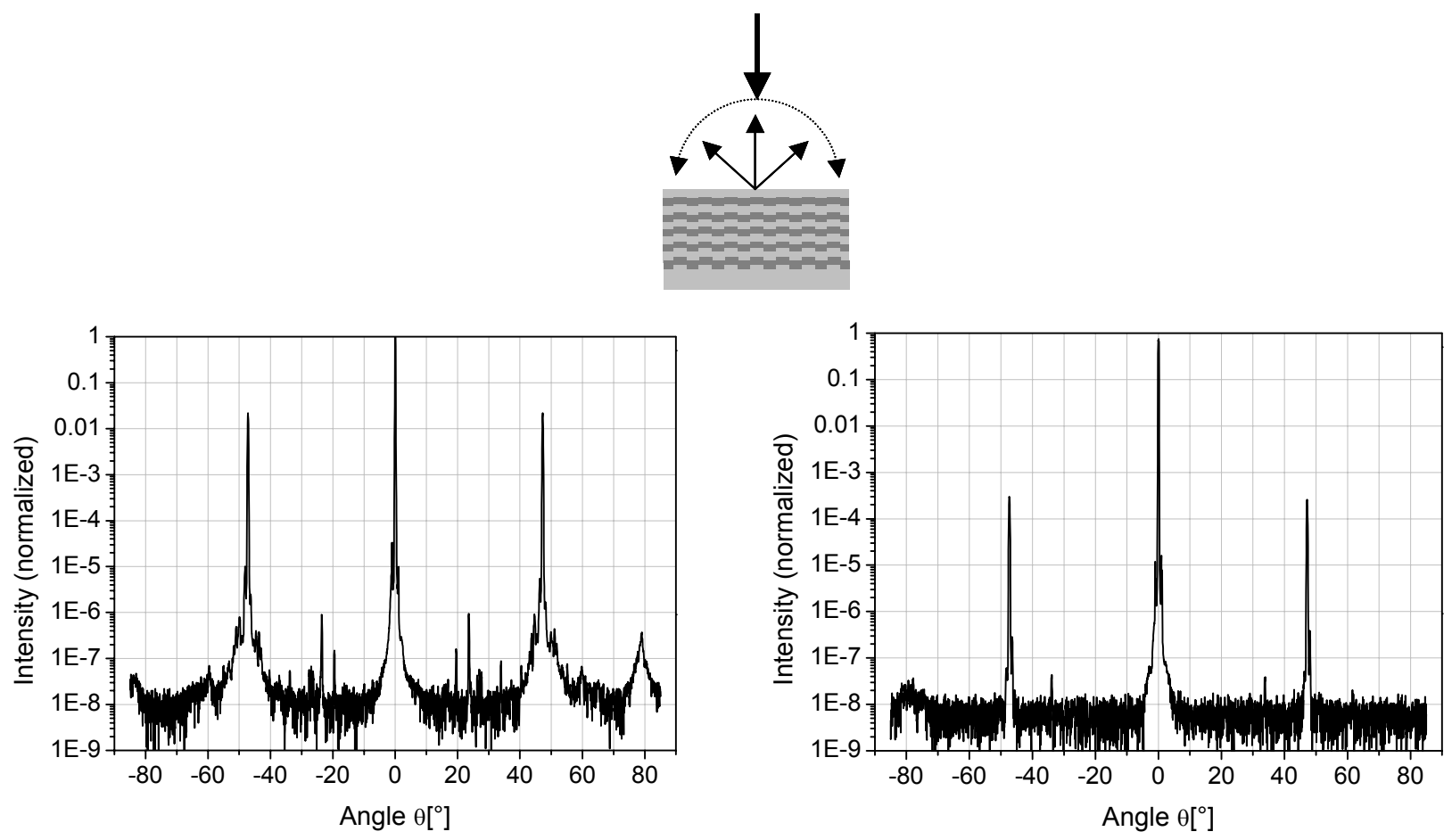

Figure 6: ARS measurement $(\lambda=1064 \mathrm{~nm})$ of coated gratings with (a) fill factor 0.49

(b) fill factor 0.67

\section{COMBINATION OF BOTH IDEAS}

In the previous sections, it has been shown that both ideas - the grating-on-top and the grating-beneath layout - have their advantages. The first one is easier to design and the reflectivity of the layerstack is not disturbed by the grating, while the second one allows for the realization of very low diffraction efficiencies and scattering losses are effectively suppressed. So it suggests itself to combine both ideas. The substrate is covered firstly by the highly-reflective stack. Afterwards, a shallow grating is generated in the topmost layer of this stack. Finally, the grating is coated by a dielectric layer of the same material as where the grating was etched into. This approach combines -at least in principle- the advantages of both layouts. The diffraction properties of the uncoated grating can be calculated like in section 3 . If the coating of the final layer is conformal, the groove depth and the general profile of the grating does not change. The diffraction efficiencies of the final device can be read in Fig. 2a by increasing the thickness of the remaining layer (how it was termed there). This means the working point shifts in the horizontal direction to the right. Fig. 7 shows the calculation of the $-1^{\text {st }}$ order diffraction efficiency $\eta_{1}$ along this line for several groove depths $(7 \mathrm{a}: 50 \mathrm{~nm}, 7 \mathrm{~b}: 100 \mathrm{~nm}$ and $7 \mathrm{c}: 290 \mathrm{~nm}$ (horizontal arrows in Fig. 2)). If the coating is non-conformal, the groove depth is decreased more and more. The shift in Fig. 2a is therefore not longer horizontal, but it has a tilt towards the $\mathrm{x}$-axis. If the uncoated grating is, for instance, $100 \mathrm{~nm}$ deep the diffraction efficiency as a function of the coating thickness starts from Fig. $7 \mathrm{~b}$ and approaches Fig. 7a. Furthermore the profile shape becomes smoother, so it changes from binary to nearly sinusoidal. The grey line in Fig. 7 shows the same calculations for a sinusoidal profile. The behavior with increasing coating thickness is similar but the efficiencies are lower. These considerations can be utilized to tune the efficiencies of the gratings. For example the grating realized in section 3 has been etched to a groove depth of $290 \mathrm{~nm}$. Since a fused silica layer with a thickness of $590 \mathrm{~nm}$ has been coated on the mirror-stack, the thickness of the residual layer beneath the grating is $300 \mathrm{~nm}$. This point is marked by a dashed line in Fig. 7c. By adding fused silica layer with a thickness of 10nm the profile shape will barely change. However, the diffraction efficiency changes from $1.5 \%$ to $2.5 \%$. A layer thickness of $20 \mathrm{~nm}$ will cause an efficiency of $3.5 \%$ and $50 \mathrm{~nm}$ will result in $8.5 \%$.

On the other hand, the coating of a further layer can be used to smooth a profile while the layerstack is undisturbed. However, to achieve an appreciable smoothing the coated layer thickness has to be at least in the range of $1 \mu \mathrm{m}$. In Fig.7, 
it can be seen that a coating of such a thickness causes a strong change of the diffraction efficiency with repeated minima and maxima. In order to maintain the desired diffraction efficiency, the layer thickness has to be chosen properly (in contrary to section 4, where a thicker layer always decreases the diffraction efficiency). To achieve the same diffraction efficiency, the grating of section 3 has to be coated by a $1 \mu \mathrm{m}$-thick layer of $\mathrm{SiO}_{2}$. The tolerance for this coating is quite small: if the coated layer was $10 \mathrm{~nm}$ to thin (this corresponds to an accuracy of $10 \%$ ), the diffraction efficiency would be only $0.5 \%$. If it was $10 \mathrm{~nm}$ to thick, $\eta_{1}$ would be increased to $3 \%$. However, for some applications, not a precise diffraction efficiency is obliged but the losses are most important, so this procedure could be an alternative.
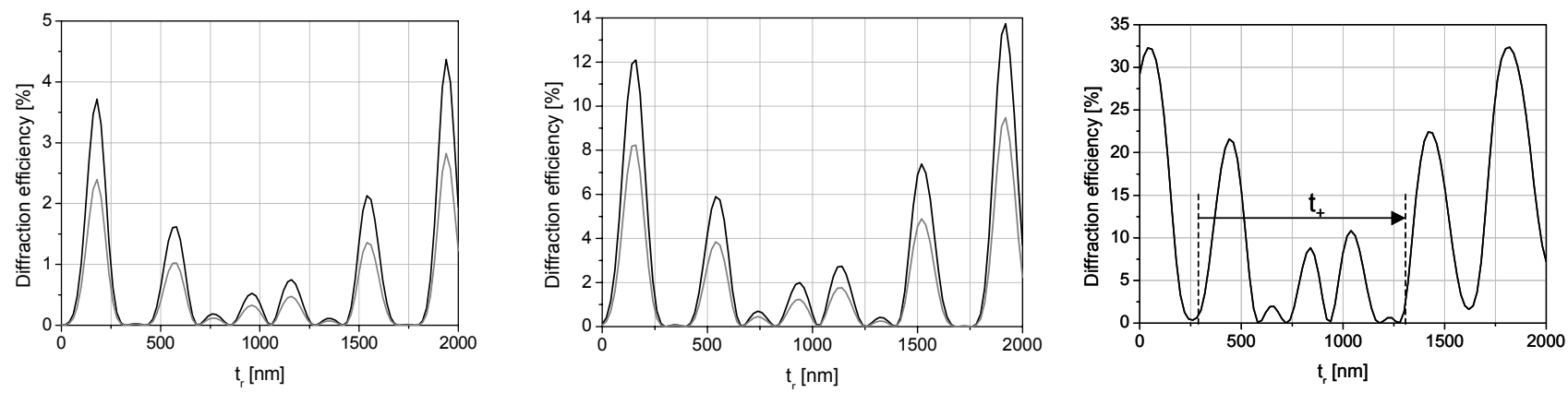

Figure 7: Theoretical diffraction efficiency as a function of the thickness $t_{\mathrm{r}}$ for conformal coating

(a) groove depth $\mathrm{h}=50 \mathrm{~nm}$

(b) groove depth $\mathrm{h}=100 \mathrm{~nm}$

(c) groove depth $\mathrm{h}=290 \mathrm{~nm}$

\section{CONCLUSIONS}

We have investigated the realization of a cavity coupler device composed of three components: a substrate, a grating and a highly reflective layerstack. The influence of their assembly (gratings on top of the stack and grating beneath) on the scattering and transmission losses have been investigated. By coating the multilayer on top of the grating the losses of a grating can be effectively reduced. High-frequency profile features such as roughness, sharp edges or periodical fill factor variations are decreased, as are the scattering losses. The smoothing of the surface corrugation also corresponds to a decrease in diffraction efficiency. This effect can be used to tune the diffraction efficiency. Diffraction efficiencies between $7 \%$ and $0.02 \%$ have been demonstrated with ultra low scattering losses. For a grating with $1.5 \%$ diffraction efficiency, a reduction of the angle-resolved scattering losses from $3 \cdot 10^{-8}$ to $1 \cdot 10^{-8}$ due to statistical scattering has been demonstrated, while parasitic diffraction orders have been drastically reduced. We furthermore discussed the possibility to combine both approaches - putting the grating on top of the layerstack and coat it additionally - in order to tune the diffraction efficiency, or to decrease the scattering losses due to roughness while maintaining the high reflectivity of the undisturbed mirror. The achievable accuracy of the resulting diffraction efficiency depends significantly on the accuracy of the employed coating process as well as the acceptable efficiency tolerance.

The losses of the cavity coupler device are one of the limiting factors of future gravitational wave detector concepts. Further investigations on these devices will focus on their mechanical quality factor and its behaviour while cooling the device down to cryogenic temperatures.

\section{ACKNOWLEDGEMENT}

This work was supported by the German Research Association (DFG) within the Sonderforschungsbereich TR 7 "Gravitational Wave Astronomy". The authors would like to thank Layertec Optical Coatings GmbH for the coating of the dielectric stacks and Dr. U. Hübner (IPHT Jena) for the high-resolution SEM-images. 


\section{REFERENCES}

1. T. Clausnitzer, J. Limpert, K. Zöllner, H. Zellmer, H. -J. Fuchs, E. -B. Kley, A. Tünnermann, M. Jupé, and D. Ristau, „Highly efficient transmission gratings in fused silica for CPA systems,” App. Opt. 42, 6934-6938 (2003)

2. B Willke et.al., "The GEO 600 gravitational wave detector," Class. Quantum Grav. 19, 1377-1387 (2002)

3. A. Abramovici, "LIGO: The Laser Interferometer Gravitational-Wave Observatory," Science 256, 325 (1992)

4. R. W. P. Drever, "Concepts for Extending the Ultimate Sensitivity of Interferometric Gravitational Wave Detectors Using Non-Transmissive Optics with Diffractive or Holographic Coupling," in Proceedings of Seventh Marcel Grossman Meeting on General Relativity, M. Kaiser and R. T. Jantzen, eds., 1401-1406 (1995)

5. A. Bunkowski, O. Burmeister, P. Beyersdorf, K. Danzmann, R. Schnabel, T. Clausnitzer, E. -B. Kley, A. Tünnermann, "Low-loss grating for coupling to a high-finesse cavity," Opt. Lett. 29, 2342-2344 (2004)

6. A. Bunkowski, O. Burmeister, K. Danzmann, and R. Schnabel, "Input-output relations for a three-port grating coupled Fabry-Perot cavity," Opt. Lett. 30, 1183-1185 (2005)

7. B. W. Shore, M. D. Perry, J. A. Britten, R. D. Boyd, M. D. Feit, H. T. Nguyen, R. Chow, G. E. Loomis, and Lifeng $\mathrm{Li}$, "Design of high-efficiency dielectric reflection gratings," J.Opt. Soc. Am. A 14, 1124-1136 (1997)

8. L. Li, J. Hirsh, "All-dielectric high-efficiency reflection gratings made with multilayer thin-film coatings," Opt. Lett. 20, 1349-1351 (1995)

9. G.A. Golubenko, A.S. Svakhin, V.A. Sychugov, A.V. Tishchenko, "Total reflection of light from a corrugated surface of a dielectric waveguide", Sov. J. Quantum Electron. 15, 886-887 (1985)

10. J. Turunen, "Diffraction theory of microrelief gratings," in Micro-optics, H.P. Herzig, ed. (Taylor \& Francis, Inc., 1997)

11. A. Duparré, J. Ferre-Borrull, S. Gliech, G. Notni, J. Steinert, J. M. Bennett, „Surface characterization techniques for determing the root-mean-square roughness and power spectral densities of optical components," Appl. Opt. 41, 154-171 (2002)

12. E. -B. Kley, T. Clausnitzer, M. Cumme, K. Zöllner, B. Schnabel, A. Stich,’'Investigation of Large-Area Gratings Fabricated by Ultrafast E-Beam Writing," in Advanced Optical Manufacturing and Testing Technology, L. Yang, H. M. Pollicove, Q. Xin, J. C. Wyant, eds., Proc. SPIE 4231, 116-125 (2000)

13. J. Stover, Optical Scattering - Measurement and Analysis, (SPIE, Bellingham, WA, 1995)

14. A. Duparré, "Light scattering of thin dielectric films," in Handbook of Optical Properties - Thin Films for Optical Coatings, R.E. Hummel, K.H. Guenther, eds. (CRC Press, Boca Raton, 1995)

15. J. K. Guha, J. A. Plascyk, "Low-Absorption Grating Beam Samplers," in Optical Components: Manufacture and Evaluation, D. Nicholson ed., Proc. SPIE 171, 117-124 (1979)

16. J. M. Elson, "Infrared Light Scattering from Surfaces Covered with Multiple dielectric overlayers," Appl. Opt. 16, 2872-2881 (1977) 